\title{
Delta Catenin
}

National Cancer Institute

\section{Source}

National Cancer Institute. Delta Catenin. NCI Thesaurus. Code C128319.

A catenin protein with 10 armadillo repeats involved in the regulation of cell adhesion and tissue morphogenesis. Catenin delta-1 (CTNND1) is expressed in vascular endothelium, keratinocytes and melanocytes, and catenin delta-2 (CTNND2) is expressed in brain. 